\title{
Solution to Selected Exercises
}

Solutions to selected exercises and all of the source code in the book can be downloaded from the WWW page:

http://www.svc.org.uk/cplus/cplusinfo.htm

or from the Macmillan site at:

http://www.macmillan-press.co.uk/mastering

Otherwise, by email, from the author with:

arthurceed-coll.ac.uk

or from the editor with:

w.buchanan@napier.ac.uk

The solutions and source code can also be requested by post, along with a floppy disk and a stamped addressed envelope, from:

Suzannah Tipple,

Macmillan Press Ltd,

Houndsmill,

Basingstoke,

Hampshire.

UK.

RG21 6XS. 